\title{
Experimental Study and Clinical Observation on the Improvement Effect of Lienal Polypeptide on Blood Toxicity and Immune Injury Induced by Radiotherapy
}

\author{
Alan Chu, Rui Song,, Ge Hou, Jinjin Yuan, ${ }^{1}$ Cheng Wang,, Yu Yang, ${ }^{1}$ Ning Qin, ${ }^{1}$ \\ Yaohe Liu, Bing Liang, ${ }^{2}$ Yan Zhang, ${ }^{3}$ and Zongwen Liu ${ }^{1}$
}

\begin{abstract}
Aims: To investigate the immune and gastrointestinal functional effects of lienal polypeptide (LP) treatment in tumor-bearing mice and carcinoma patients receiving radiotherapy (RT), and to detect hematological indicators and T lymphocyte subsets.

Methods: Tumor-bearing mice were randomly divided into five groups: the control group, the RT group, the RT+LP-L (1.7 mg/kg, low dosage of LP) group, the RT+LP-M $(5.2 \mathrm{mg} / \mathrm{kg}$, middle dosage of LP) group, and the $\mathrm{RT}+\mathrm{LP}-\mathrm{H}(10.4 \mathrm{mg} / \mathrm{kg}$, high dosage of LP) group. In addition, carcinoma patients were randomly divided into two groups. The observation group was given LP during RT, and the control group was only treated with RT. We then compared the myelosuppression, gastrointestinal reactions, and clinical efficacy among groups.

Results: In the animal experiments, compared with the control group, the number of leukocytes and lymphocytes of the mice in the "RT" group decreased $(p<0.05)$. Animals receiving LP evidenced a dose-response curve with regard to the number of leukocytes and lymphocytes that was proportional to the LP dose, increased $(p<0.05)$. Flow cytometric analyses showed that LP treatment of the mice increased the numbers of $\mathrm{CD}^{+}$, and $\mathrm{CD}^{+}{ }^{\mathrm{T}}$ cells and the $\mathrm{CD} 4^{+} / \mathrm{CD}^{+}$ratio. In our clinical study, the Radiation Therapy Oncology Group (RTOG)/ European Organization for Research and Treatment of Cancer (EORTC) criteria were used for measuring myelosuppression and gastrointestinal reactions. The RTOG/EORTC grade 3 or 4 inhibition rate of leukocytes, granulocytes, hemoglobin, platelets, and gastrointestinal toxic effects in the observation group were significantly lower than that in the control group $(p<0.05)$.
\end{abstract}

Conclusion: LP can improve the hematopoietic and immune function of RT-treated mice and reduce the hematological and gastrointestinal toxicity of patients treated with RT and improve the quality of life.

Keywords: carcinoma, lienal polypeptide injection, radiotherapy, mice

\section{Introduction}

C ANCER DIAGNOSES ARE increasing annually and both the cancer itself and the treatments can greatly affect the health and quality of life of the patients (Liu et al., 2019). The number of deaths due to cancer has also increased significantly for the past several years. Radiotherapy (RT) plays an important role in the treatment of malignant tumors (Ahmad et al., 2012); however, due to its toxicity many patients suffer from myelosuppression and immunosuppression, as well as experiencing adverse gastrointestinal issues. These side effects can interfere with the completion of RT and affect it efficacy (Zhou et al., 2016; Luo et al., 2018; Feng et al., 2019). In this study, lienal polypeptide (LP) was used to treat tumor-bearing mice during RT. By detecting peripheral blood and immune-related indicators, the effects of LP treatment on the immune function of tumor-bearing mice after irradiation were investigated. The effects of LP treatment during RT on hematopoietic function and gastrointestinal function in patients with malignant tumors were also investigated and discussed.

\section{Materials and Methods \\ Cell line and cell culture}

Murine sarcoma S180 cells were donated by the institute of medical sciences of Henan Province (Zhengzhou University). The S180 cells were cultured in the peritoneal cavity of mice and subcultured using ascites fluid.

Departments of ${ }^{1}$ Radiotherapy and ${ }^{2}$ Oncology, The Second Affiliated Hospital of Zhengzhou University, Zhengzhou, China.

${ }^{3}$ Department of Pharmacology, School of Basic Medical of Zhengzhou University, Zhengzhou, China. 
Tumor-bearing mouse model

and administration method

ICR mice (16-22 g, 6-8 weeks old, half were female and half male) were purchased from Hunan Slake Jingda Experimental Animal Co., Ltd. LP was provided by Jilin Fengsheng Pharmaceutical Co., Ltd. S180 cells $\left(5 \times 10^{6}\right.$ cells per mouse) were injected in the axilla of the right forearm. When the tumor volume $\left[=(\text { long diameter } \times \text { short diameter })^{2}\right]$ reached $500 \mathrm{~mm}^{3}$, the mice were randomly divided into one of five groups: a control group, an RT group, an RT combined with low-dose $(1.7 \mathrm{mg} / \mathrm{kg})$ LP group (RT+LP-L), an RT combined with medium-dose $(5.2 \mathrm{mg} / \mathrm{kg}) \mathrm{LP}$ group (RT+LP-M), and an RT combined with high-dose $(10.4 \mathrm{mg} / \mathrm{kg}) \mathrm{LP}$ group (RT+LP$\mathrm{H})$. There were eight mice in each group.

\section{Radiotherapy method}

Irradiation (Linear Accelerato, Elekta Synergy) was performed on the axilla of the solid tumor of tumor-bearing mice, once a day at a dose of 2 Gy for five consecutive days. Normal saline was injected into the tail vein of the control group and the RT-only group daily. The earlier-indicated concentrations $(1.7,5.2$, and $10.4 \mathrm{mg} / \mathrm{kg})$ of LP in saline were, respectively, injected into the tail veins of the three drug groups for the five consecutive days of RT. The RT methods and the doses of LP for the mice were calculated based on the methods used in the clinic.

\section{Peripheral blood cell detection}

On the fifth day of treatment, $20 \mu \mathrm{L}$ of orbital blood was collected from each mouse and was diluted with $180 \mu \mathrm{L}$ of diluent [Sysmex Medical Electronics (Shanghai) Co., Ltd., Shanghai]. The numbers of leukocytes, neutrophils, red blood cells, hemoglobin, platelets, and lymphocytes were assessed using a blood cell analyzer [Sysmex Medical Electronics (Shanghai) Co., Ltd.].

\section{Analysis of lymphocyte subsets}

On the fifth day after the initiation of treatment, $100 \mu \mathrm{L}$ of orbital blood was collected from each mouse and added to a heparin anticoagulant tube. Next, $500 \mu \mathrm{L}$ of cell lysate (Guangzhou Zhaokang Biological Technology Co., Ltd., Guangzhou) was added to each tube, shaken and mixed for $5 \mathrm{~min}$, and centrifuged at $1500 \mathrm{~g}$ for $5 \mathrm{~min}$ at $4^{\circ} \mathrm{C}$. The resulting supernatant was discarded and the pellet was resuspended with

Table 1. Number of Leukocytes, Lymphocytes, and Red Blood Cells in Peripheral Blood OF MicE $(\overline{\mathrm{x}} \pm \mathrm{s}, N=8)$

\begin{tabular}{lccr}
\hline Group & $\begin{array}{c}\text { Hemameba } \\
\left(\times 10^{9} / L\right)\end{array}$ & $\begin{array}{c}\text { Lymphocyte } \\
\left(\times 10^{9} / L\right)\end{array}$ & \multicolumn{1}{c}{$\begin{array}{c}\text { Erythrocyte } \\
\left(\times 10^{12} / L\right)\end{array}$} \\
\hline Model & $14.47 \pm 4.48$ & $11.12 \pm 3.54$ & $11.57 \pm 6.20$ \\
RT & $10.47 \pm 5.58^{*}$ & $6.65 \pm 3.60^{*}$ & $8.64 \pm 0.74$ \\
RT+LP-L & $7.64 \pm 1.30$ & $7.16 \pm 3.29$ & $8.99 \pm 1.58$ \\
RT+LP-M & $11.38 \pm 2.03$ & $8.10 \pm 1.53$ & $10.09 \pm 1.45$ \\
RT+LP-H & $12.04 \pm 1.20^{\#}$ & $8.98 \pm 1.45$ & $11.30 \pm 4.81$ \\
\hline
\end{tabular}

*Compared with the model group, $p<0.05$; " compared with radiotherapy combined with low-dose lienal polypeptide group, $p<0.05$.

LP, lienal polypeptide; RT, radiotherapy.
Table 2. Peripheral Blood Hemoglobin, Platelets, and Neutrophil Number of Mice $(\overline{\mathrm{x}} \pm \mathrm{s}, N=8)$

\begin{tabular}{lccc}
\hline Group & $\begin{array}{c}\text { Hemoglobin } \\
(\mathrm{g} / \mathrm{L})\end{array}$ & $\begin{array}{c}\text { Platelet } \\
\left(\times 10^{9} / \mathrm{L}\right)\end{array}$ & $\begin{array}{c}\text { Neutrophil } \\
\left(\times 10^{9} / \mathrm{L}\right)\end{array}$ \\
\hline Model & $192 \pm 113$ & $790 \pm 584$ & $3.30 \pm 1.71$ \\
RT & $191 \pm 79$ & $854 \pm 451$ & $3.45 \pm 3.63$ \\
RT+LP-L & $129 \pm 33$ & $663 \pm 601$ & $4.51 \pm 2.19$ \\
RT+LP-M & $161 \pm 48$ & $419 \pm 323$ & $3.41 \pm 2.19$ \\
RT+LP-H & $161 \pm 25$ & $630 \pm 285$ & $3.04 \pm 1.06$ \\
\hline
\end{tabular}

a pipette gun in $100 \mu \mathrm{L}$ phosphate-buffered saline (PBS). Then anti-CD8-phycoerythrin monoclonal antibody (eBioscience), anti-CD4-FITC monoclonal antibody (eBioscience), and antiCD3-allophycocyanin (APC)-APC monoclonal antibody (eBioscience) were added, respectively, for flow cytometric analyses (Becton, Dickinson and Company). This was followed by a 30 min incubation in the dark in a $4^{\circ} \mathrm{C}$ ice bath. The samples were then centrifuged at $1500 \mathrm{~g}$ for $5 \mathrm{~min}$ at $4^{\circ} \mathrm{C}$, and the supernatant was again discarded. The precipitated cells were resuspended and mixed with $500 \mu \mathrm{L}$ PBS. The $\mathrm{T}$ cell subtype numbers were determined using BectonDickson (BD) FACS and analyzed with the BD Accuri C6 Software (Becton Dickinson and Company).

\section{Patients}

From November 2015 to November 2018, 150 patients with lung cancer, esophageal cancer, cervical cancer, rectal cancer, and gliomas were enrolled from the Second Affiliated Hospital of Zhengzhou University. This study was approved by the Ethics Committee of Second Affiliated Hospital of Zhengzhou University. Written informed consent was obtained from all the patients. All subjects were divided into two groups according to a random number table, 75 were assigned to the observation group and 75 to the control group. The ratio of men to women in the observation group was $37: 38$, with an average age of $47.35 \pm 1.86$ years. The ratio of men to women in the control group was $36: 39$, with an average age of $49.28 \pm 2.04$ years. There were no statistically significant differences between the two groups in terms of age and gender $(p>0.05)$. Patients with the same type of tumor were identical in staging and treatment.

\section{Treatment}

The control group received only RT when clinically indicated. The observation group received RT combined with LP. The dose of RT for all patients was provided as intensive-

Table 3. Analysis of T Lymphocyte Subsets in Orbital Blood of Mice in Each Group $(\overline{\mathrm{X}} \pm \mathrm{s}, N=8)$

\begin{tabular}{llll}
\hline Group & \multicolumn{1}{c}{$C D 3^{+}(\%)$} & \multicolumn{1}{c}{$C D 4^{+}(\%)$} & $C D 4^{+} / C D 8^{+}$ \\
\hline Model & $41.13 \pm 12.93$ & $22.02 \pm 11.02$ & $1.52 \pm 0.58$ \\
RT & $21.66 \pm 6.87^{*}$ & $10.60 \pm 5.03^{*}$ & $0.88 \pm 0.29^{*}$ \\
RT+LP-L & $25.60 \pm 4.44$ & $13.81 \pm 7.24$ & $1.05 \pm 0.62$ \\
RT+LP-M & $27.06 \pm 12.13$ & $15.90 \pm 6.38$ & $1.22 \pm 0.65$ \\
RT+LP-H & $32.86 \pm 14.35^{\#}$ & $21.80 \pm 12.54^{\#}$ & $1.49 \pm 0.65^{\#}$ \\
\hline
\end{tabular}

*Compared with the model group, $p<0.05$; "compared with radiotherapy alone, $p<0.05$. 
TABle 4. Comparison of Leukocytes, Granulocytes, Hemoglobin, and Platelet Between the Two Groups Before Treatment $(\overline{\mathrm{X}} \pm \mathrm{s}, N=75)$

\begin{tabular}{lcccc}
\hline Group & $\begin{array}{c}\text { Leukocyte } \\
\left(\times 10^{9} / \mathrm{L}\right)\end{array}$ & $\begin{array}{c}\text { Granulocyte } \\
\left(\times 10^{9} / \mathrm{L}\right)\end{array}$ & $\begin{array}{c}\text { Hemoglobin } \\
(\mathrm{g} / \mathrm{L})\end{array}$ & $\begin{array}{c}\text { Platelet } \\
\left(\times 10^{9} / \mathrm{L}\right)\end{array}$ \\
\hline $\begin{array}{c}\text { Observation } \\
\text { group }\end{array}$ & $5.1 \pm 3.05$ & $3.13 \pm 2.75$ & $115 \pm 18$ & $194 \pm 101$ \\
$\begin{array}{c}\text { Control } \\
\text { group }\end{array}$ & $6.0 \pm 3.67$ & $4.09 \pm 3.37$ & $119 \pm 18$ & $222 \pm 82$ \\
\hline
\end{tabular}

modulated RT (Linear Accelerato, Elekta Synergy), $6 \mathrm{mV} \mathrm{X-}$ ray, a radiation dose of 50-60 Gy/25-30 times, divided into seven or nine fields, 1.8-2.0 Gy/time, five times per week, with a total course of treatment of 5-6 weeks.

For the observation group LP was administered through an intravenous drip at a concentration of $8 \mathrm{mg} / 100 \mathrm{~mL}$ prepared in sterile physiological saline.

\section{White blood cell analyses}

The number of peripheral blood cell types, including total leukocytes, neutrophils, lymphocyte subtypes, RBCs and platelets of patients in the two groups, was determined the week before RT and after the last dose of RT. The degree of inhibition was analyzed. Gastrointestinal adverse reactions of patients were monitored before and after RT, and the degree of adverse reactions was analyzed.

\section{Grading criteria for myelosuppression and gastrointestinal reactions}

The Radiation Therapy Oncology Group (RTOG) and European Organization for Research and Treatment of Cancer (EORTC) standards were used as references (Cox et al., 1995).

For a comparison of the short-term efficacy of the RT between the two patient groups before and after treatment, a CT examination was used to observe the local control of the tumor. According to response evaluation criteria in solid tumors (RECIST), complete responses (CR) and partial responses (PR) were effective, and the rest were ineffective. The short-term response rate $(\%)(\mathrm{RR})=(\mathrm{CR}+\mathrm{PR}) /$ total number of patients $\times 100 \%$.

\section{Statistics}

SPSS17.0 software was used for the statistical analyses. The number of peripheral blood cells and $\mathrm{T}$ lymphocyte subsets were represented by a mean \pm standard deviation $(\overline{\mathrm{x}} \pm \mathrm{s})$ and analyzed using one-way ANOVA. The rates of RTOG/EORTC grade 3 or 4 bone marrow inhibition (myelosuppression), and the rates of RTOG/EORTC grade 3 or 4 gastrointestinal side effects were measured using the chi-square test, values of $p<0.05$ were considered statistically significant.

\section{Results}

\section{Murine experimental results}

Compared with the control group, the number of leukocytes and lymphocytes in the blood of the mice in the RT group was decreased, and the difference was statistically significant $(p<0.05)$. Those animals treated with LP and RT cohorts displayed increased numbers of leukocytes and lymphocytes compared with the controls and there was a doseresponse based on the amount of LP administered. Compared with the "RT+LP-L," the leukocyte number of the mice in the "RT+LP-H" increased significantly $(p<0.05)$. Moreover, the "RT+LP-H" cohort mice displayed the greatest improvement in the number of erythrocytes, the number of neutrophils, and the number of platelets and the hemoglobin concentration compared with the RT-only group; however, the results were not significant $(p>0.05)$. However, as can be seen in Table 2 the erythrocyte number in the RT-only group was lower than that in all of the LP-treatment groups, and the value gradually increased with the increasing doses of LP (Tables 1 and 2).

Flow cytometry was used to analyze the T lymphocyte subsets derived from murine orbital blood specimens. Compared with the control group, the ratios of $\mathrm{CD}^{+}, \mathrm{CD}^{+}$, and $\mathrm{CD} 4^{+} / \mathrm{CD}^{+}{ }^{+} \mathrm{T}$ lymphocyte subsets of the "RT-only" group all decreased, and the differences were statistically significant $(p<0.05)$. Compared with the RT group, the ratios of $\mathrm{CD}^{+}, \mathrm{CD}^{+}$, and $\mathrm{CD}^{+} / \mathrm{CD}^{+}$in the peripheral blood of the "RT+LP-H" group were restored, and the differences were statistically significant $(p<0.05)$ (Table 3$)$.

\section{Clinical trial results}

There were no significant differences in the number of leukocytes, granulocytes, hemoglobin, and platelets between the observation group and the control group before treatment $(p>0.05)$, as shown in Table 4. Post-treatment, the number of patients with RTOG/EORTC grade 3 or 4 inhibition of leukocytes, granulocytes, hemoglobin, and platelets in the observation group were significantly lower than those in the control group, and the difference was statistically significant $(p<0.05)$, as shown in Table 5.

Table 5. Comparison of Myelosuppression in Both Groups After Treatment $(N=75)$

\begin{tabular}{llcccccc}
\hline Group & Project & O (Case) & I (Case) & II (Case) & III (Case) & IV (Case) & III-IV Inhibition rate (\%) \\
\hline Observation & Hemameba & 15 & 27 & 25 & 6 & 2 & $8(10.7)^{*}$ \\
& Granulocyte & 16 & 28 & 24 & 5 & 2 & $7(9.3)^{*}$ \\
& Hemoglobin & 26 & 34 & 14 & 1 & 0 & $1(1.3)^{*}$ \\
\multirow{5}{*}{ Control } & Platelet & 30 & 24 & 19 & 2 & 0 & $2(2.7)^{*}$ \\
& Hemameba & 10 & 9 & 33 & 19 & 4 & $23(30.7)$ \\
& Granulocyte & 10 & 8 & 34 & 19 & 4 & $23(30.7)$ \\
& Hemoglobin & 9 & 23 & 35 & 6 & 2 & $8(10.7)$ \\
& Platelet & 6 & 20 & 40 & 8 & 1 & $9(12)$ \\
\hline
\end{tabular}

*Compared with the control group, $p<0.05$. 
Table 6. Comparison of the Incidence of Gastrointestinal Adverse Reactions After Treatment Between the Two Groups $(N=75)$

\begin{tabular}{lcccccc}
\hline Group & $O($ Case $)$ & $I$ (Case) & II (Case) & III (Case) & IV (Case) & III-IV Inhibition rate (\%) \\
\hline Observation & 10 & 40 & 23 & 2 & 0 & $2(2.7)^{*}$ \\
control & 5 & 26 & 35 & 7 & 2 & $9(12)$
\end{tabular}

*Compared with the control group, $p<0.05$.

The RTOG/EORTC grade 3 or 4 incidence of gastrointestinal adverse reactions in the observation group was also significantly lower than that in the control group, and the difference was statistically significant $(p<0.05)$, as shown in Table 6 .

The short-term efficacy of the RT for the two patients groups was evaluated by RECIST criteria $(86.7 \%$ in the observation group and $81.3 \%$ in the control group), and it was found that in terms of objective effective rate, that there was no statistically significant difference $(p>0.05)$, as shown in Table 7 .

\section{Discussion}

Exposure to carcinogenic factors is increasingly resulting in increased incidences of malignant tumors in the human population. The number of deaths due to cancer has also increased year by year. As one of the three major methods for the treatment of malignant tumors, RT has garnered significant attention, and its position in clinical work has gradually become more prominent. A large proportion of patients with malignant tumors can be treated through radiation therapy that not only kills tumor cells but also damages normal tissue cells, particularly those that undergo rapid proliferation such as epithelial cells and blood cells (Kang et al., 2018). Radiotherapy for thoracic, abdominal, and pelvic tumors often causes gastrointestinal side effects resulting in discomfort and sometimes the necessity to delay or stop treatment. Therefore, the prevention and treatment of adverse reactions during $\mathrm{RT}$ in patients with malignant tumors and alleviating the suffering of patients are major challenges faced by radiotherapists and radiation oncologists.

LP is a polypeptide with a molecular weight $<6000 \mathrm{Da}$. It is provided as sterile solution that is free of free amino acids, nucleic acid, and sugar. It is extracted from the spleen of healthy calves and has been shown to stimulate the proliferation of bone marrow cells, resulting in an increase in the number of leukocytes, and generally improving hematopoietic function. It has also been shown to reduce the toxic effects of radiation and chemotherapy (Xie and Wang, 2018; Xia et al., 2018; Yang, 2018). In our animal model experiments, RTtreated animals when compared with the untreated control group displayed a decreased number of leukocytes and lymphocytes in their orbital blood. This result indicates that RT can damage the hematopoietic system of mice and decrease the hematopoietic function of bone marrow. However, the application of LP coincident with RT helped to normalize the numbers of leukocyte and lymphocytes in a dose-dependent manner. Although there was no statistical difference in the erythrocyte numbers between the groups, we observed that the erythrocyte number in the "RT" group decreased when compared with the model group, and the erythrocyte number trended upward with increasing concentrations of LP. We also observed that the hemoglobin concentration and platelet numbers of the "RT+LP" groups were not significantly improved compared with the "RT" group. The reason may be that the hemoglobin and platelets have a long metabolic cycle.

In this study, the flow cytometric results showed that compared with the control murine group, the $\mathrm{CD}^{+}, \mathrm{CD}^{+}$, and $\mathrm{CD}^{+} / \mathrm{CD}^{+}$ratios of the $\mathrm{T}$ cell subsets of mice in the "RT" were decreased, indicating that RT could damage the immune system of mice. The "RT+LP-H" compared with the "RT," the ratio of $\mathrm{CD}^{+}, \mathrm{CD}^{+}$, and $\mathrm{CD} 4^{+} / \mathrm{CD}^{+}$were restored, and this suggests that the LP can improve the immune damage induced by RT in mice. This result is consistent with a previous study by $\mathrm{Wu}$ et al. (2018). In this case the authors found that cyclophosphamide (CTX) treatment significantly lowered the number of $\mathrm{CD}^{+} \mathrm{CD}^{+}$and $\mathrm{CD} 3^{+} \mathrm{CD} 8^{+} \mathrm{T}$ lymphocytes and decreased the $\mathrm{CD}^{+} \mathrm{CD}^{+} / \mathrm{CD}^{+} \mathrm{CD}^{+}$ratio. These indices were significantly improved by LP treatment. In a study of RT for esophageal cancer patients, Song et al. (2016) found that LP combined with radiotherapy produced a significant shortterm increase in the efficacy of treatment for advanced esophageal cancer. Zeng et al. (2017) also studied the application of LP during RT in patients with esophageal cancer, and found that the incidences of esophageal, hepatic, and renal dysfunction were significantly reduced during RT with LP in patients with advanced esophageal cancer. These authors also noted that the number of T lymphocyte subsets in patients with LP changed significantly. Unfortunately, the effect of LP on T lymphocyte subsets in patients was not examined in this study. In the future, we will explore the improvements of the immune system of tumor patients during RT by LP.

When Zhou et al. (2016) studied the hematological toxicity of postoperative chemotherapy for colon cancer patients, they found that the incidence of myelosuppression was

Table 7. Comparison of Short-Term Efficacy Between Observation Group and Control Group

\begin{tabular}{lccccc}
\hline & \multicolumn{3}{c}{ The recent curative effect } \\
\cline { 2 - 5 } Group & $C R$ (case) & $P R$ (case) & SD (case) & PD (case) & Near-term efficiency, $\mathrm{n}(\%)$ \\
\hline Observation $(n=75)$ & 10 & 55 & 9 & 1 & $65(86.7)$ \\
control $(n=75)$ & 10 & 51 & 12 & $61(81.3)$ & 0.79 \\
$\chi^{2}$ & & & & 0.37 \\
$p$ & & & & \\
\hline
\end{tabular}

$\mathrm{CR}$, complete responses; PR, partial responses; SD, standard deviation. 
significantly reduced when chemotherapy was combined with LP. The average Karnofsky (KPS) score of the patients in the LP group was significantly increased. In our study, compared with the "RT" group, we found that the incidence of the RTOG/EORTC grade 3 or 4 myelosuppression was significantly reduced after treatment with LP, and thus, we show that LP can reduce the side effects of RT in patients with malignant tumors. This finding is consistent with previous reports (Li et al., 2019).

Numerous studies have shown that LP can improve the immune function of cancer patients, alleviate myelosuppression, and improve the quality of life; however, research on clinical efficacy is inconsistent. Liu and Pan (2016) found that LP injections can improve the objective efficiency of breast cancer patients treated with letrozole. Han et al. (2016a) combined LP with chemotherapy for ovarian cancer patients and found that the incidence of myelosuppression in patients with tumors above grade II decreased, which was consistent with the results of our study. Subsequently, Han et al. (2016b) also studied cervical cancer patients and found that the incidence of myelosuppression in patients with early cervical cancer was reduced when combined with LP during neoadjuvant chemotherapy, but there was still no significant difference in the short-term effective rate between the two groups.

In summary, LP treatment was shown to improve the hematopoietic function and immune function of RT-treated mice, and to reduce the hematological toxicity and gastrointestinal reactions of RT patients and improve their quality of life.

\section{Authors' Contributions}

All authors participated in the design, interpretation of the studies and analysis of the data, and review of the article. A.C., Y.Y., N.Q., and Y.L. conceived and designed the experiments; R.S., G.H., J.Y., C.W., and B.L. performed the experiments; Z.L. and Y.Z. administered and supervised the project, and acquired funding; A.C. wrote the original article; Z.L. reviewed and edited the article.

\section{Author Disclosure Statement}

No competing financial interests exist.

\section{Funding Information}

This study was supported by the First Batch of Science and Technology Plan Projects of Zhengzhou in 2013 (No. 131PCXTD628) and the Medical Science and Technology Plan Program Grant of Henan Province (No. 201401009).

\section{References}

Ahmad SS, Duke S, Jena R, et al. (2012) Advances in radiotherapy. BMJ 345:e7765.

Cox JD, Stetz J, Pajak TF (1995) Toxicity criteria of the Radiation Therapy Oncology Group (RTOG) and the European Organization for Research and Treatment of Cancer (EORTC). Int J Radiat Oncol Biol Phys 31:1341-1346.

Feng J, Cheng X, Zeng H, et al. (2019) Clinical research on stereotactic radiosurgery combined with epithermal growth factor tyrosine kinase inhibitors in the treatment of brain metastasis of non-small cell lung cancer. J BUON 24:578-584.

Han H, Wang J, Li YL, et al. (2016a) Influence of the lienal polypeptide injection combined with chemotherapy on clini- cal effect and immune function in advanced ovarian cancer. Chin J Clin Rat Drug Use 9:33-35.

Han H, Wei Q, Qiu G, et al. (2016b) Effect and clinical efficacy of lienal polypeptide injection on immune function of cervical cancer patients with neoadjuvant chemotherapy. J Hebei Med Univ 37:1424-1427.

Kang M, Wang F, Liao X, et al. (2018) Intensity-modulated radiotherapy combined with endostar has similar efficacy but weaker acute adverse reactions than IMRT combined with chemotherapy in the treatment of locally advanced nasopharyngeal carcinoma. Medicine (Baltimore) 97:e11118.

Li J, Wang K, Li W, et al. (2019) Clinical observation of lienal polypeptide-assisted letrozole in the treatment of hormone receptor-positive breast cancer in the elderly. Chin Clin Oncol 24:163-166.

Liu X, Yu C, Bi Y, et al. (2019) Trends and age-period-cohort effect on incidence and mortality of prostate cancer from 1990 to 2017 in China. Public Health 172:70-80.

Liu ZW, Pan YY (2016) Clinical trial of lienal polypeptide injection combined with letrozole tablets in the treatment of hormone receptor positive elderly advanced breast cancer. Chin J Clin Pharmacol 32:2135-2137, 2151.

Luo HC, Lin GS, Liao SG, et al. (2018) Cervical cancer treated with reduced-volume intensity-modulated radiation therapy base on Sedlis criteria (NCCN VS RTOG). Br J Radiol 91: 20170398.

Song XL, Shi XY, Wen YF, et al. (2016) Effect of spleen polypeptide combined with radiotherapy on the short-term efficacy of advanced esophageal carcinoma and its influence on the level of T lymphocyte subsets 15:939-942.

Wu YP, Deng J, Ouyang SH, et al. (2018) Immune regulation effect of lienal polypeptides extract in Lewis lung carcinomabearing mice treated with cyclophosphamide. Exp Biol Med (Maywood) 243:66-77.

Xia WJ, Yu RB, Jiang BS, et al. (2018) A clinical observation of spleen polypeptide injection combined with concurrent chemoradiotherapy for limited-stage small cell lung cancer. J Nanjing Med Univ (Nat Sci) 38:356-359.

Xie J, Wang ZM (2018) Effect of spleen polypeptide injection on myelosuppression and $\mathrm{T}$ lymphocyte subsets in patients with lung cancer after chemotherapy. Chin J Gerontol 38: 5450-5451.

Yang JH (2018) Application effects of Lienal polypeptide injection on postoperative adjuvant chemotherapy in patients with non-small cell lung cancer. Med J Chin Peoples Health 30:19-21.

Zeng XW, Lv HS, Gao DS, et al. (2017) Therapeutic effect of spleen polypeptide combined with radiotherapy for advanced esophageal cancer. Pract Clin Med 18:19-20, 23.

Zhou J, Niu G, Pei Y, et al. (2016) The effect and clinical efficacy of lienal polypeptide injection combined with FOLFOX chemotherapy regimen in colon cancer patients. Oncol Lett 12:3191-3194.

Address correspondence to:

Zongwen Liu

Department of Radiotherapy

The Second Affiliated Hospital of Zhengzhou University Zhengzhou

Henan 450014

China

E-mail: liuzwhh@sina.com 